\title{
Miniature Fresnel LC Lens Performance
}

\section{Dieter Cuypers $^{1}$, Rik Verplancke ${ }^{1}$, and Herbert De Smet ${ }^{1}$}

\author{
dieter.cuypers@ugent.be \\ ${ }^{1}$ Centre for Microsystems Technology, imec and Ghent University, Technologiepark 126, B-9052 Ghent, \\ Belgium \\ Keywords: liquid crystal; tunable lens; Fresnel
}

\begin{abstract}
The performance of miniature, polymer-based Fresnel liquid crystal lenses is discussed, specifically the sensitivity for non-uniformities.
\end{abstract}

\section{INTRODUCTION}

\subsection{Fresnel liquid crystal lenses}

Applications that feature miniature lenses, with diameters around $10 \mathrm{~mm}$ or less are nowadays abundant and include smartphones, medical use instruments but also high-end uses such as intra-ocular lenses or wearables like contact lenses. The small diameter in combination with the typically desired focal lengths such systems result in relatively slow lenses, with working $\mathrm{f}-$ numbers in the range of 10 or more. The lens design that best exploits the high f-number is the segmented Fresnel lens. In essence, these are sectioned conventional lenses relying entirely on refraction, thus giving very simple lens designs that can still achieve high optical quality even when liquid crystal is the optical medium [1].

To fully exploit one of the key benefits of a Fresnel lens, its low profile height, it is ideally made up of thin, polymer layers, ensuring the total lens thickness can remain as limited as a few hundred micrometers. Thus, a typical switchable liquid crystal lens consists of a polymer foil on which the Fresnel segments are imprinted in a UV-curable material [2], with nematic, non-twisted liquid crystal on top and a second foil to seal the cell.

In figure 1, the working principle is illustrated once more for clarity. Assuming planar alignment, in the unpowered state a mismatch between the ordinary refractive index of the LC and the index of the lens shape material will give the intended focusing power. With an electric field applied, the liquid crystal molecules will gradually reorient themselves to the vertical position. As such, the mismatch of the refractive indies will reduce until, with correctly selected materials the extraordinary index of the liquid crystal equals the index of the lens material and refraction no longer occurs.

\section{PERFORMANCE}

Previously, it has been shown that the liquid crystal based Fresnel lens can perform quite well in the intended environments, especially considering that it basically a single lens [3]. This is illustrated in Figure 2, showing the MTF graph for a representative lens.
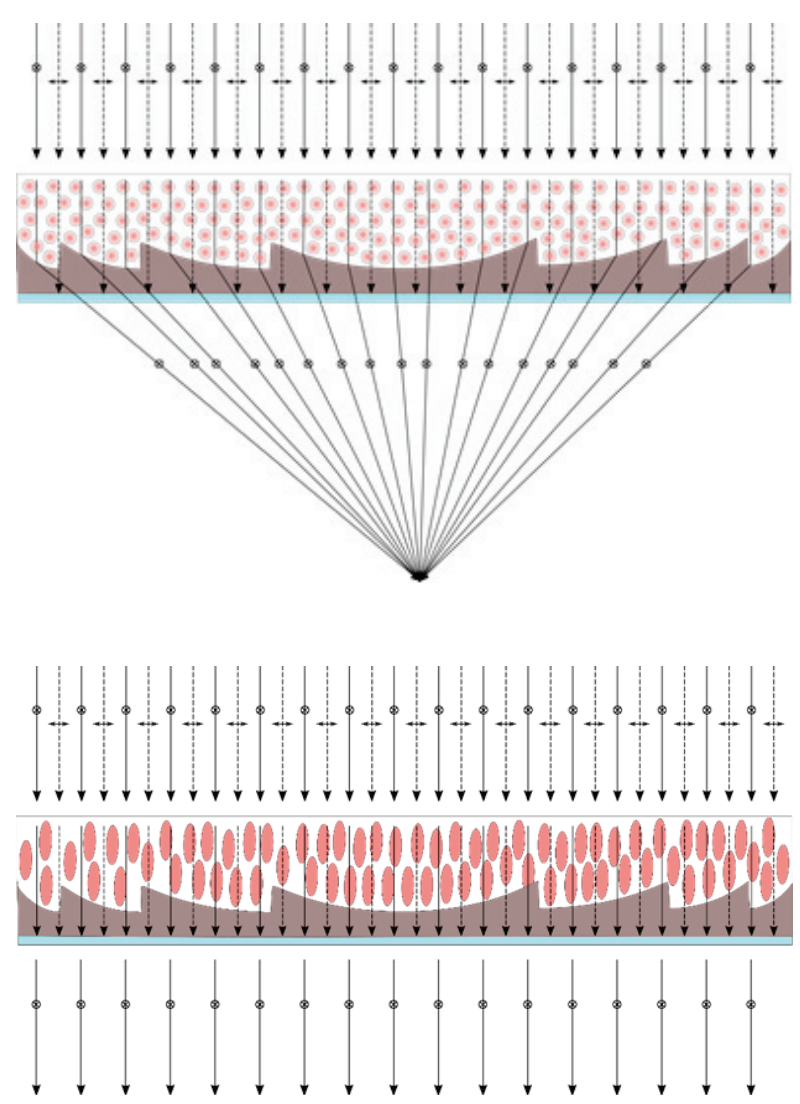

Fig. 1 Basic liquid crystal Fresnel lens working principle.

In the unpowered state (top) the LC molecules are aligned planar. The mismatch between the extraordinary refractive index of the LC and the index of the lens material will then cause refraction to the focal point for one polarization, the other is blocked by the polarizer (light blue) beneath the lens. When a voltage is applied (bottom) the LC molecules reorient to the vertical position. If the ordinary refractive index of the LC and the index of the lens material have been chosen to match, no refraction occurs and the light simply passes through. 


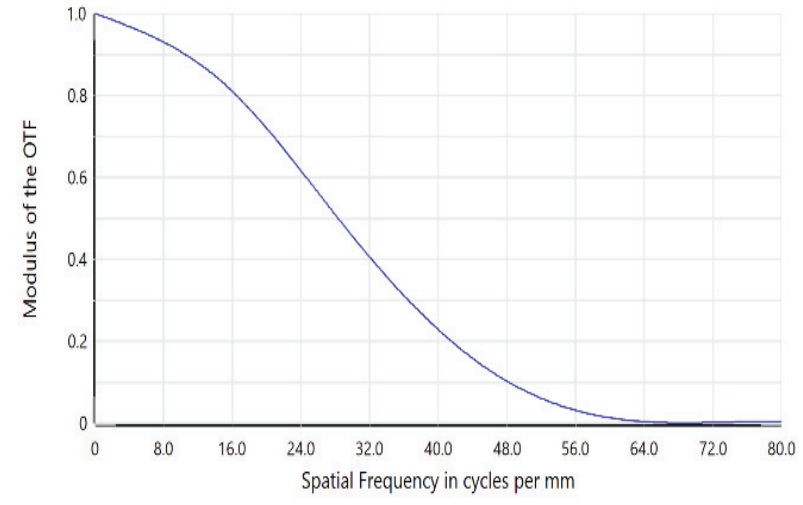

Figure 2: Calculated Modulation Transfer Function for a nominal $125 \mathrm{~mm}$ focal distance lens.

These performance levels are however assuming a perfect manufacturing process and disregard any inevitable tolerances that will be incurred. Specifically for the process, the use of thin foil materials makes the lens susceptible to mismatches between the intended local liquid crystal thickness and the real values. Figure 3 shows a typical height profile of the base lens material throughout a cross-sectioned Fresnel lens.

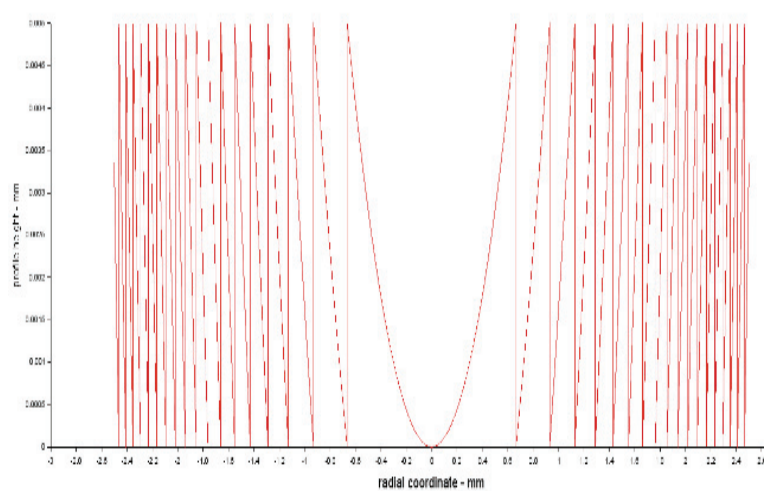

Figure 3: Typical height profile of a miniature Fresnel lens.

Calculated constant height profile with $5 \mu \mathrm{m}$ blazes.

The liquid crystal thickness on top of it is determined by the distance to the top foil, set by a spacer technology. Deviations, most likely caused by depressions in the top foil then result in local aberrations of the optical power. Studying the profile, it can be seen that sensitivity for these deviations will be largest in the center part of the lens, where the sag of the lens changes very gradually $(5 \mu \mathrm{m}$ over a distance of more than $600 \mu \mathrm{m})$. Hence, even small depressions in the order of $100 \mathrm{~nm}$ can cause noticeable optical phenomena in that area.

Figure 4 illustrates this by means of image quality prediction for a concentric depression. Obviously, the optical impact can be tremendous.

\section{CONCLUSIONS}

Fresnel type liquid crystal lenses made of soft, flexible materials need adequate gap spacing control to achieve maximum performance.

\section{REFERENCES}

[1] D. Cuypers, "Design of Active Liquid Crystal Based Contact Lenses", SID 2019 Digest, pp. 985 (2019)

[2] X. Shang, J.-Y. Tan, J. De Smet, P. Joshi, E. Islamaj, D. Cuypers, M. Vervaeke, J. Van Erps, H. Thienpont H. De Smet, "Replicating micro-optical structures using soft embossing technique", Proc. SPIE 9231, 30th European Mask and Lithography Conference, 92310H (2014)

[3] D. Cuypers, R. Verplancke and H. De Smet, "Miniature Liquid Crystal Lens Optimizations", IDW 19, pp 1360-1363 (2019)
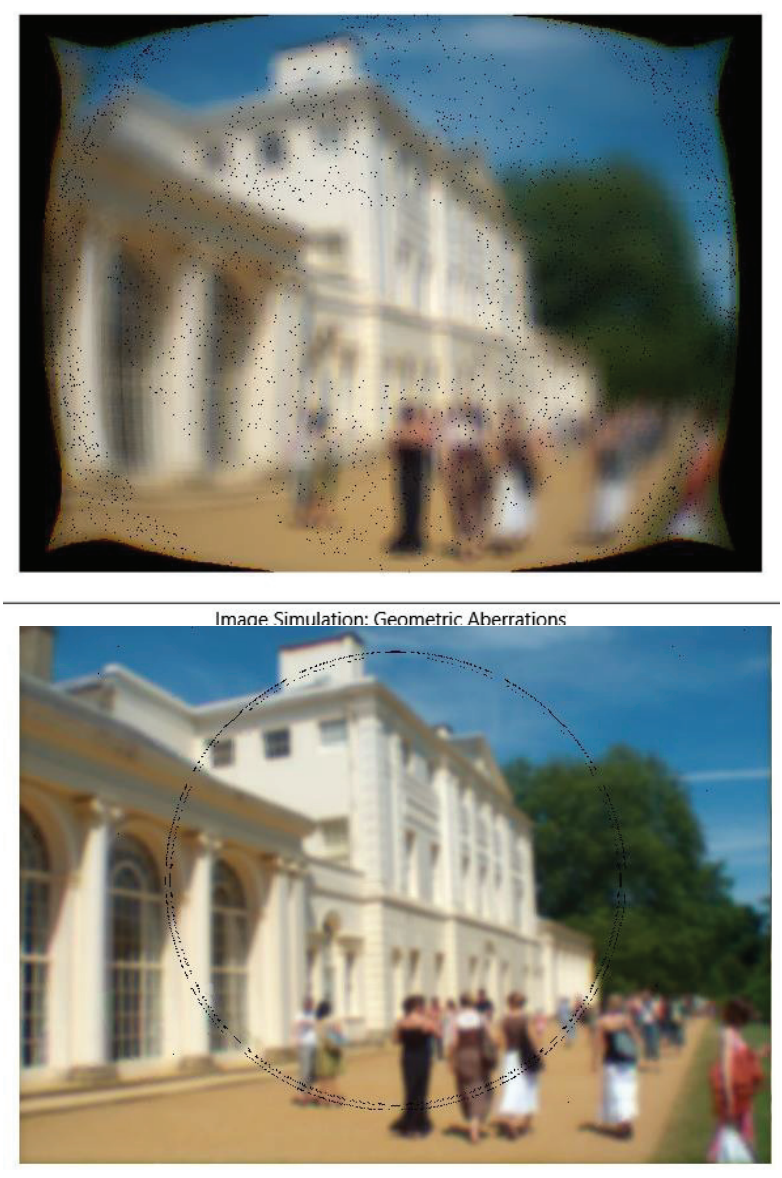

Imaqe Simulation: Geometric Aberrations

Figure 4: Simulated image quality of a Fresnel LC lens.

Top with an assumed local concentric depression in the liquid crystal thickness, bottom with the intended values. 